\title{
Free energy calculations for atomic solids through the Einstein crystal/molecule methodology using GROMACS and LAMMPS
}

\author{
J.L. Aragones, C. Valeriani and C. Vega \\ Departamento de Química Física, Facultad de Ciencias Químicas, \\ Universidad Complutense de Madrid, 28040 Madrid, Spain
}

(Dated: October 16, 2012)

\begin{abstract}
In this work the free energy of solid phases is computed for the Lennard-Jones potential and for a model of $\mathrm{NaCl}$. The free energy is evaluated through the Einstein crystal/molecule methodologies using the Molecular Dynamics programs: GROMACS and LAMMPS. The obtained results are compared with the results obtained from Monte Carlo. Good agreement between the different programs and methodologies was found. The procedure to perform the free energy calculations for the solid phase in the Molecular Dynamic programs is described. Since these programs allow to study any continuous intermolecular potential (when given in a tabulated form) this work shows that for isotropic potentials (describing for instance atomic solids or colloidal particles) free energy calculations can be performed on a routinely basis using GROMACS and/or LAMMPS.
\end{abstract}

In 1984 Frenkel and Ladd proposed the "Einstein crystal method", a novel scheme to compute the free energy of a solid [1]: the method is based on a thermodynamic integration of the Helmholtz free energy in the canonical ensemble along a reversible path between the system of interest and an ideal Einstein crystal with the same structure as the real solid, whose Helmholtz free energy can be analytically computed. In the ideal Einstein crystal, particles are attached to their lattice positions via harmonic springs (of constant $\Lambda_{E}$ ). More recently, some of us have proposed the "Einstein molecule method" [2, 3], a variant of the Einstein crystal to compute the free energy of molecular solids. The main difference between both methods is the choice of the reference system. In the Einstein crystal the reference system is an ideal Einstein crystal with the constraint of the center-of-mass of the system kept fixed (to avoid a quasi-divergence of the integral of the free energy change from the reference system to the real solid). In the Einstein molecule the reference system is an ideal Einstein crystal with the constraint of the position of one particle kept fixed.

The Helmholtz free energy $A_{\text {sol }}(T, V)$ computed with the Einstein crystal/molecule calculations can be written as $A_{\text {sol }}(T, V)=A_{0}(T, V)+\Delta A_{1}(T, V)+\Delta A_{2}(T, V)$, where $\mathrm{A}_{0}$ is the free energy of the reference system (including corrections for the fixed point), whose analytical expression is slightly different in the Einstein crystal and Einstein molecule, $\Delta \mathrm{A}_{1}$ is the free energy difference between the ideal Einstein crystal and the Einstein crystal in which particles interact through the Hamiltonian of the real solid ("interacting" Einstein crystal) and $\Delta \mathrm{A}_{2}$ is the free energy difference between the interacting Einstein crystal and the real solid [4]. The expression for both $\Delta \mathrm{A}_{1}$ and $\Delta \mathrm{A}_{2}$ is the same in both methods although its value may be different since the fixed point (centerof-mass or a reference particle) is different (a detailed description of these terms is provided as Supplementary Material[5]). In any case since the free energy of a solid is uniquely defined, its value should not depend on the methodology used to compute it [2, 4] .

Besides the Einstein crystal methods other methods have been developed in the last decade to estimate the free energy of solids (or to determine the fluid-solid equilibria), such as the Phase Switch method [6] and an integration path to calculate the Gibbs free energy difference between any arbitrary solid and liquid proposed by Grochola [7]. In spite of this progress the number of groups performing free energy calculations for solids is still small [8 10]. Solid free energy calculations can be easily adapted into Monte Carlo scheme, but it requires one to write a la carte MC codes. Therefore it seems of interest to consider if these calculations can be implemented in widely used open source Molecular Dynamics simulation programs such as GROMACS [11] or LAMMPS [12]. As far as we are aware, free energy calculations of solids are not yet explicitly implemented in such packages and only recently free energy calculations for alloys of copper and zirconium [13] using LAMMPS through the Einstein crystal method have been reported. Since GROMACS and/or LAMMPS incorporate the possibility of using harmonic potentials restraining the position of atoms (or even freezing the position of atoms) and incorporate thermostats that can correctly treat harmonic oscillators (as for instance the new thermostat of Bussi et al.[14]), it seems possible to perform Einstein crystal/molecule calculations for atomic solids using these programs. In this note we shall use GROMACS and LAMMPS to compute the free energy of solids of particles interacting via isotropic potentials using either the Einstein crystal or the Einstein molecule method. In particular we will compute the free energy of Lennard-Jones (LJ) and of Sodium Chloride modeled via the Joung-Cheatham(JC)-NaCl potential [15] (using the $\mathrm{SPC} / \mathrm{E}$ set) which describes ion-ion interactions through a LJ potential plus a Coulomb term. To confirm the results obtained with GROMACS (G) and LAMMPS (L), calculations will be also performed using a Monte Carlo code (MC).

We have carried out free energy calculations for a 256 LJ-Argon spherically truncated and shifted (STS) at $r_{c}=2.7 \sigma$ and for a $1372 \mathrm{LJ}$ spherically truncated (ST) at $r_{c}=5 \sigma$. Results of the free energy calculations 


\begin{tabular}{|c|c|c|c|c|c|c|c|}
\hline & & $\mathrm{N}$ & $\mathrm{r}_{c}$ & $A_{0}$ & $\Delta A_{1}$ & $\Delta A_{2}$ & $A_{\text {sol }}$ \\
\hline \multicolumn{8}{|l|}{ EC } \\
\hline $\mathrm{MC}$ & LJ/STS & 256 & $2.7 \sigma$ & 13.61 & $-3.14(1)$ & $-7.36(3)$ & $3.11(4)$ \\
\hline G & LJ/STS & 256 & $2.7 \sigma$ & 13.61 & $-3.14(1)$ & $-7.35(3)$ & $3.10(4)$ \\
\hline $\mathrm{L}$ & LJ/STS & 256 & $2.7 \sigma$ & 13.61 & $-3.14(1)$ & $-7.36(3)$ & $3.11(4)$ \\
\hline $\mathrm{MC}$ & $\mathrm{LJ} / \mathrm{ST}$ & 1372 & $5 \sigma$ & 13.68 & $-3.69(1)$ & $-7.40(3)$ & $2.59(4)$ \\
\hline G & $\mathrm{LJ} / \mathrm{ST}$ & 1372 & $5 \sigma$ & 13.68 & $-3.69(1)$ & $-7.38(3)$ & $2.61(4)$ \\
\hline $\mathrm{MC}$ & $\mathrm{JC}-\mathrm{NaCl}$ & 1000 & $14 \AA$ & 10.70 & $-159.94(1)$ & $-6.34(4)$ & $-155.58(5)$ \\
\hline G & $\mathrm{JC}-\mathrm{NaCl}$ & 1000 & $14 \AA$ & 10.70 & $-159.95(1)$ & $-6.30(4)$ & $-155.55(5)$ \\
\hline \multicolumn{8}{|l|}{ EM } \\
\hline $\mathrm{MC}$ & LJ/STS & 256 & $2.7 \sigma$ & 13.64 & $-3.14(1)$ & $-7.39(3)$ & $3.11(4)$ \\
\hline G & LJ/STS & 256 & $2.7 \sigma$ & 13.64 & $-3.14(1)$ & $-7.38(3)$ & $3.12(4)$ \\
\hline $\mathrm{L}$ & LJ/STS & 256 & $2.7 \sigma$ & 13.64 & $-3.15(1)$ & $-7.40(3)$ & $3.09(4)$ \\
\hline $\mathrm{MC}$ & $\mathrm{LJ} / \mathrm{ST}$ & 1372 & $5 \sigma$ & 13.69 & $-3.69(1)$ & $-7.40(3)$ & $2.60(4)$ \\
\hline G & $\mathrm{LJ} / \mathrm{ST}$ & 1372 & $5 \sigma$ & 13.69 & $-3.69(1)$ & $-7.39(3)$ & $2.61(4)$ \\
\hline $\mathrm{MC}$ & $\mathrm{JC}-\mathrm{NaCl}$ & 1000 & $14 \AA$ & 10.71 & $-159.95(1)$ & $-6.35(4)$ & $-155.59(5)$ \\
\hline G & $\mathrm{JC}-\mathrm{NaCl}$ & 1000 & $14 \AA$ & 10.71 & $-159.95(1)$ & $-6.34(4)$ & $-155.57(5)$ \\
\hline
\end{tabular}

TABLE I. Free-energies of solids as obtained from Einstein Crystal (EC) and Einstein Molecule (EM) methodologies using $\mathrm{MC}$ or $\mathrm{MD}(\mathrm{G} ; \mathrm{L}) . \mathrm{N}$ is the number of particles, $r_{c}$ is the cut-off distance of the LJ contribution. Results for the LJ/STS and LJ/ST systems were obtained at $T^{*}=k_{B} T / \epsilon=2$ and $\rho^{*}=\rho \sigma^{3}=1.28$ for the fcc structure. Results for the $\mathrm{NaCl}$ solid were obtained for the JC-NaCl model at $T=298 K$ and a volume of $V=24.13 \mathrm{~nm}^{3}$. Free energies are in $N k_{B} T$ units.

are presented in Table [. For the LJ/STS system $\mathrm{A}_{0}, \Delta$ $\mathrm{A}_{1}$, and $\Delta \mathrm{A}_{2}$ obtained from $\mathrm{MC}$ and from MD (GROMACS/LAMMPS) are in very good agreement, with a free energy difference lower than $0.03 N k_{B} T$ (typical uncertainties in calculations of free energy of solids being of $\left.0.05 N k_{B} T\right)$. The choice of a LJ spherically truncated and shifted (STS) avoids the subtle issues arising when comparing results obtained by $\mathrm{MC}$ and $\mathrm{MD}$ for a spherically truncated (ST) potential[5, 16, 17]. In the LJ/ST truncated at $r_{c}=5 \sigma$, the free energy results obtained with MC and MD agree quite well with each other and with previous calculations for the same system size and thermodynamic state [2, 18, 19]. It is clear that the magnitude of problems arising with the discontinuity of the potential at the cut-off are also quite small when the cut- off distance is large enough $(\approx 5 \sigma)$.

Notice that although for a certain system size and Hamiltonian the computed free energy of a solid is unique, in general the free energy of solids changes with the system size. As a suggestion, in order to have a reasonable estimate of the free energy of a solid, we recommend to use a relatively large system size (with more than 1000 molecules) and to average properties over around $10^{4}-10^{5}$ independent configurations for evaluating $\Delta A_{1}$ and runs of about $10 \mathrm{~ns}$ for $\Delta A_{2}$. Moreover, the use of a large cut-off (above $4.5 \sigma$ ) is recommended. In all LJ systems, both Einstein molecule and Einstein crystal give the same value of the free energy $A_{\text {sol }}$, although the single contributions are slightly different in both methodologies (as they should be). In the Supplementary Material, we provide further details on the implementation 5], needed for GROMACS and LAMMPS to easily compute $\mathrm{A}_{0}, \Delta$ $\mathrm{A}_{1}$, and $\Delta \mathrm{A}_{2}$.

To show that the methodology also works for more complex systems where particles interact via an isotropic potential, we calculate the free energy of $\mathrm{NaCl}$ using the Joung-Cheatham potential [15]. Results are presented in Table I. We simulate a 1000 ions $\mathrm{NaCl}$ solid (at $298 \mathrm{~K}$ and the equilibrium density of the model at 1 bar). The free energies computed from MC and GROMACS agree reasonably well (the difference been of $0.03 N k_{B} T$ ) and are in good agreement with previous calculations [20].

To conclude, we have demonstrated that it is possible to compute the free energy of atomic solids using GROMACS and LAMMPS for systems interacting with spherical potentials such as Lennard-Jones, Yukawa, Morse and any continuous potential since both packages incorporate the possibility of using a tabulated numerical intermolecular potentials. We do hope that this work stimulate more groups to perform free energy calculations for solids. For future work it would be useful to analyze if for molecular fluids the Einstein crystal/molecule calculations could also be performed with GROMACS and/or LAMPPS.

This work was funded by the grants FIS2010-16159 (DGI) and MODELICO-P2009/ESP/1691 (CAM). J.L. Aragones acknowledges MEC for a pre-doctoral grant. C. Valeriani acknowledges financial support from a Juan de la Cierva Fellowship and from the Marie Curie Integration Grant PCIG-GA-2011-303941 ANISOKINEQ.
[1] D. Frenkel and A. J. C. Ladd, J. Chem. Phys. 81, 3188 (1984).

[2] C. Vega and E. G. Noya, J. Chem. Phys. 127, 154113 (2007).

[3] E. G. Noya, M. M. Conde, and C. Vega, J. Chem. Phys. 129, 104704 (2008).

[4] C. Vega, E. Sanz, E. G. Noya, and J. L. F. Abascal, J. Phys. Condens. Matter 20, 153101 (2008).

[5] See EPAPS Document No. E-JCPS for further details about how to implement the free energy calculations in GROMACS and LAMMPS including input files.

[6] A. D. Bruce, N. B. Wilding, and G. J. Ackland, Phys. Rev. Lett. 79(16), 3002 (1997).

[7] G. Grochola, J. Chem. Phys. 120, 2122 (2004).

[8] J. Anwar, D. Frenkel, and M. G. Noro, J. Chem. Phys. 118, 728 (2003).

[9] P. A. Monson and D. A. Kofke, in Advances in Chemical Physics, edited by I. Prigogine and S. A. Rice (John Wiley and Sons, 2000), vol. 115, p. 113. 
[10] A. P. Hynninen, M. E. Leunissen, A. van Blaaderen, and M. Dijkstra, Phys. Rev. Lett. 96, 018303 (2006).

[11] D. van der Spoel, E. Lindahl, B. Hess, G. Groenhof, A. E. Mark, and H. J. C. Berendsen, J. Comput. Chem. 26, 1701 (2005).

[12] S. Plimpton, J. Comp. Phys. 117, 1 (1995).

[13] C. Tang and P. Harrowell, J. Phys. Condens. Matter 24, 245102 (2012).

[14] G. Bussi, D. Donadio, and M. Parrinello, J. Chem. Phys. 126, 014101 (2007).

[15] I. Joung and T. Cheatham, J. Phys. Chem. B 112, 9020 (2008).
[16] E. de Miguel and G. Jackson, J. Chem. Phys. 125, 164109 (2006).

[17] D. Frenkel and B. Smit, Understanding Molecular Simulation (Academic Press, London, 2002).

[18] M. A. Barroso and A. L. Ferreira, J. Chem. Phys. 116(16), 7145 (2002).

[19] L. Yakub and E. Yakub, J. Chem. Phys. 136, 144508 (2012).

[20] J. L. Aragones, E. Sanz, and C. Vega, J. Chem. Phys. 136, 244508 (2012). 